\title{
Necessidade de fertilizante e calcário em área sob sistema plantio direto considerando variabilidade espacial ${ }^{1}$
}

\author{
Pedro H. Weirich Neto ${ }^{2}$, Cláudio B. Sverzut ${ }^{3}$ \& Adriana Schimandeiro ${ }^{4}$
}

\begin{abstract}
RESUMO
A região dos Campos Gerais do Paraná é pioneira no sistema plantio direto, apresentando rendimentos elevados e alta tecnificação. Neste sentido, surgem técnicas para auxiliar na otimização desses processos, a exemplo da agricultura de precisão ou manejo localizado de solo e culturas. Com este trabalho, objetivou-se avaliar as necessidades localizadas de fertilizante e calcário em lavoura comercial sob sistema plantio direto e comparar os resultados com a recomendação tradicional. As amostras de solo foram coletadas em malha retangular, formada por quatro linhas de quinze pontos eqüidistantes 40 m, totalizando 60 pontos (9,6 ha). Os dados de capacidade de troca catiônica (CTC), saturação por bases (V\%), fósforo (P) e potássio (K) foram analisados pela estatística descritiva e pela geoestatística. Foi possível observar grandes diferenças nas necessidades de nutrientes e de calagem. Consideraram-se apenas fórmulas NPK comercialmente disponíveis. Conforme a recomendação tradicional para a área em estudo, deixou-se de aplicar $175 \mathrm{~kg}$ de $\mathrm{P}_{2} \mathrm{O}_{5}$ e se aplicaram 138,9 kg de $\mathrm{K}_{2} \mathrm{O}$ a mais que o requerido. A necessidade de calagem entre os 60 pontos variou de zero a $4 \mathrm{Mg} \mathrm{ha}^{-1}$, totalizando $7,84 \mathrm{Mg}$. Sugere-se um estudo econômico mais detalhado para uma possível adoção pelo produtor.
\end{abstract}

Palavras-chave: agricultura de precisão, taxa variável de reposição, fertilidade do solo

\section{Fertilizer and limestone requirement in an area under no tillage system considering spatial variability}

\begin{abstract}
The Campos Gerais region, in the State of Paraná, Brazil, is a pioneer in no tillage system, presenting high yields and high technical standards. Techniques such as precision farming or site-specific management have emerged to assist on the optimization of this process. The objective of this research was to evaluate the localized requirements of fertilizer and limestone in a commercial field under no tillage system and compare results with the traditional recommendation. The soil samples were collected in a grid formed by four rows of fifteen points each, $40 \mathrm{~m}$ distant of each other, in a total of 60 points (9.6 ha). Results for cation exchange capacity (CEC), sum of bases ( $\mathrm{V} \%$ ), phosphorous (P) and potassium (K) were analyzed by exploratory data analysis and geostatistics. Large differences in nutrient and liming needs were observed. Only commercially available NPK formulations were considered. Following traditional recommendation, $175 \mathrm{~kg}$ of $\mathrm{P}_{2} \mathrm{O}_{5}$ less and $138.9 \mathrm{~kg}$ of $\mathrm{K}_{2} \mathrm{O}$ more than required were applied for the study area. The liming necessities within the 60 points varied from zero to $4 \mathrm{Mg} \mathrm{ha}^{-1}$, totalizing $7.84 \mathrm{Mg}$. A complementary detailed economic study is suggested, for possible adoption by farmers.
\end{abstract}

Key words: precision farming, variable rate application, soil fertility

1 Trabalho extraído da Tese de Doutorado do primeiro autor

${ }^{2}$ LAMA/DESOLO/UEPG. Av. Gal. Carlos Cavalcanti 4748. CEP 84030-900, Ponta Grossa, PR. Fone (42) 3220-3092. E-mail: lama1@uepg.br

${ }_{3}^{3}$ FEAGRI/UNICAMP; Cidade Universitária Zeferino Vaz, CP 6011. CEP 13083-875, Campinas, SP. Fone (19) 3788-1051. Email: bianor@agr.unicamp.br

${ }^{4}$ Mestranda em Manejo Sustentável de Recursos. Universidade Tecnológica de Munique. Frankendorf 12, Buttenhein, 96155, Alemanha. Fone (+ 49/9545) 922-336.

E-mail: aschima1@yahoo.com.br 


\section{INTRODUÇÃO}

A região dos Campos Gerais, no Estado do Paraná, possui características próprias dentro da agricultura brasileira. Pioneira no sistema Plantio Direto, apresenta alta tecnificação e rendimentos elevados. A SEAB (2003) relata um rendimento médio de milho para a referida região de $6,8 \mathrm{Mg} \mathrm{ha}^{-1}$. Com o objetivo de obter altos rendimentos, o processo produtivo deve respeitar algumas variáveis, desde a implantação da cultura, passando pelo processo de semeadura, propriedades do solo, condução fitotécnica e fitopatológica, até a colheita. Neste sentido, surgem técnicas para auxiliar na otimização desses processos, a exemplo da agricultura de precisão, ou manejo localizado das culturas.

De acordo com Balastreire (1997), a agricultura de precisão não é uma técnica nova. Elaborado em 1920 por pesquisadores americanos que estudavam a necessidade localizada de calcário, o conceito foi utilizado por algum tempo e depois abandonado, quando surgiram, então os equipamentos de tração mecânica. Reapareceu com o crescimento da consciência ambiental, segundo Goering (1993), tendo em vista que as aplicações localizadas diminuem as chances de escape e contaminação por produtos químicos.

Visando a um aproveitamento melhor dos recursos agrícolas, muitos trabalhos sobre manejo localizado de culturas estão sendo desenvolvidos, especialmente em relação à aplicação localizada de nutrientes no solo. De acordo com Malzer (1996), os métodos da agricultura de precisão podem ser utilizados com qualquer variável agrícola, apesar de sua origem ser o manejo de nutrientes e fertilidade do solo. Como afirmam Tweeten (1996) e Balastreire (1997), o impacto ambiental causado pela distribuição excessiva de fertilizantes é uma das razões para esses estudos, em virtude de problemas de lixiviação dos nutrientes, atingindo o lençol freático, e de carreação pelas chuvas, poluindo rios, córregos e lagos. Outra razão é o prejuízo econômico causado pela aplicação de fertilizante em excesso em alguns pontos e insuficiente em outros, diminuindo a produção, devido ao método convencional utilizado no cálculo de adubação, onde se realiza apenas uma amostragem representativa para a análise química de uma área, obtendo-se um valor médio de aplicação.

O crescimento das plantas, conforme a lei do mínimo, citada por Mello (1983), Mengel \& Kirkby (1987) e van Raij (1991), é limitado pelo nutriente menos disponível no solo. Os aumentos sucessivos nas quantidades disponíveis no solo, em função de utilização de quantidades crescentes de fertilizantes, também podem atingir níveis prejudiciais ao crescimento das plantas. Este é o princípio da lei dos incrementos decrescentes. Ndiaye \& Yost (1989), em estudo sobre potássio na cultura do milho observaram que, em áreas onde havia menor variação na concentração deste nutriente, o rendimento médio da cultura atingia níveis superiores.

Reichardt et al. (1986) afirmam que duas formas principais de amostragem experimental podem ser utilizadas: a inteiramente casualizada, na qual cada observação é independente das demais, e a regionalizada, em que a coleta é feita de acordo com um plano espacial determinado. Teorias como a geoestatística, disponibilizada por Matheron (1963), podem ser empregadas no caso de amostragem regionalizada, tendo como resultado a descrição da variabilidade espacial, a partir de correlogramas e semivariogramas, que definem o grau de dependência no espaço da grandeza medida e o alcance de cada amostragem. Conforme Cahn et al. (1994) a geoestatística consiste de análise variográfica e krigagem. A análise variográfica utiliza o semivariograma para modelar a variância espacial dos dados, ao passo que a krigagem usa a variância modelada para estimar (inferir) valores.

Assim, Souza et al. (1998) observaram correlação espacial cruzada positiva entre o rendimento de trigo e as variáveis fósforo, potássio e matéria orgânica, sob o sistema Plantio Direto. Vieira (1997), em estudo sobre a variabilidade espacial de atributos químicos e físicos de uma área experimental sob sistema convencional, constatou haver coeficientes de variação diferentes para as camadas do solo de $1-25 \mathrm{~cm}$ e $25-50 \mathrm{~cm}$, sendo os maiores coeficientes na primeira camada. Observou, também, dependência espacial apenas entre os atributos da primeira camada e variabilidades semelhantes.

O IPF (1998) mapeou o solo de uma área considerada homogênea, comparando aplicação generalizada e aplicação específica de fertilizantes. Constatou-se que o manejo localizado representou aumento nos custos com fertilizantes, porém concentrados em locais necessários. Sá (2001), em trabalho semelhante, relata que não houve diferença nos custos com fertilizantes, mas apenas uma distribuição espacial diferenciada.

Objetivou-se, com o presente trabalho, avaliar as necessidades localizadas de fertilizante e calcário em uma lavoura comercial, sob o sistema plantio direto; compará-las com o método convencional de estimativa e quantificar as eventuais perdas, considerando-se fórmulas de adubo comercialmente disponíveis.

\section{MATERIAL E MÉTODOS}

Este trabalho foi realizado no ano agrícola 1999/2000, pelo grupo de trabalho do Laboratório de Mecanização Agrícola da Universidade Estadual de Ponta Grossa (LAMA/ UEPG), em uma área de lavoura comercial na região dos Campos Gerais, PR, localizada em latitude $25^{\circ} 01^{\prime} 14$ ' S e longitude $50^{\circ} 18^{\prime} 42^{\prime \prime} \mathrm{O}$. É típico desta região rochas do tipo folhelho e siltitos com intercalações de arenitos (Godoy, 1991). Na área em estudo o solo foi classificado como Latossolo Vermelho-Amarelo Distrófico. O solo apresentava um horizonte B latossólico, isto é sem diferenças significativas entre o conteúdo de argila no horizonte A e B, valor do conteúdo de argila no horizonte $\mathrm{B}$, para cinco amostras, foi de $490 \mathrm{~g} \mathrm{~kg}^{-1}$ (EMBRAPA, 1999).

O talhão utilizado possuía área total aproximada de 22 ha, se encontrava há doze anos sob o sistema plantio direto, sendo a rotação de culturas utilizada nos últimos anos: soja ( $G l y$ cine max) (1997/1998), triticale (X Triticosecale Widdmark) (1998), soja (1998/1999), aveia preta (Avena strigosa) (sem exportação de grãos) (1999) e milho (Zea mays) na safra agrícola 1999/2000, época de condução do experimento. 
Coletaram-se amostras de solo para análise em malha retangular, formada por quatro linhas de quinze pontos eqüidistantes $40 \mathrm{~m}$, totalizando 60 pontos, em uma área aproximada de 9,6 ha. Retiraram-se seis subamostras para compor cada amostra.

Para a recomendação tradicional de fertilizantes e calcário, conforme metodologia do agrônomo responsável, realizou-se também a amostragem convencional, isto é, uma única amostra formada por várias subamostras em caminhamento zigue-zague no talhão.

As análises químicas básicas dos 60 pontos foram realizadas no Laboratório de Fertilidade do Solo da UEPG. Os valores obtidos de fósforo (por Mehlich), potássio, capacidade de troca catiônica (CTC) e saturação por bases (V\%), foram utilizados para o cálculo da necessidade de calagem, da fórmula NPK comercialmente disponível específica para cada ponto, e da diferença entre o fertilizante aplicado e o específico para cada ponto.

Os dados foram analisados sob a estatística descritiva pela distribuição de freqüência, coeficiente de variação, valores de assimetria e curtose; em seguida, realizou-se a análise geoestatística para verificar a variabilidade espacial entre as amostras, por meio da distribuição da variância e do semivariograma. Para confeccionar os mapas da área em estudo utilizou-se o programa computacional Surfer ${ }^{\circledR}$ (Golden Software Inc.) e na obtenção dos semivariogramas utilizouse o programa Variowin (Pannatier, 1996).

\section{RESULTADOS E DISCUSSÃO}

De posse dos resultados da análise da amostragem convencional (Tabela 1), o agrônomo responsável recomendou, para a referida área, $260 \mathrm{~kg} \mathrm{ha}^{-1}$ da fórmula NPK 14-18-15 e $0,5 \% \mathrm{Zn}$ como adubação de base e $110 \mathrm{~kg} \mathrm{ha}^{-1}$ da fórmula NPK 36-00-12 em cobertura, visando à instalação da cultura do milho.

Os valores obtidos pela estatística descritiva dos dados de CTC, V\%, fósforo e potássio, são apresentados na Tabela 2 e na Figura 1. Avaliando-se, pelo método descrito por Jones (1969), apenas os dados de CTC e V\% podem ser considerados como de distribuição normal, com base nos valores de assimetria e curtose. As distribuições de potássio e fósforo apresentaram-se assimétricas indicando, talvez, a existência de divisões na área de estudo.

Tabela 2. Estatística descritiva dos 60 dados de potássio, capacidade de troca catiônica, fósforo e saturação por bases

\begin{tabular}{|c|c|c|c|c|}
\hline & K & CTC & \multirow{2}{*}{$\underset{\mathrm{mg} \mathrm{dm}}{P}$} & \multirow{2}{*}{$\begin{array}{l}\text { V } \\
\%\end{array}$} \\
\hline & \multicolumn{2}{|c|}{$\mathrm{cmol}_{\mathrm{c}} \mathrm{dm}^{-3}$} & & \\
\hline Média & 0,20 & 13,30 & 6,10 & 61,10 \\
\hline Desvio Padrão & 0,11 & 0,95 & 2,35 & 8,14 \\
\hline CV (\%) & 50,36 & 7,20 & 38,53 & 13,33 \\
\hline Simetria & 1,07 & $-0,31$ & 0,95 & $-0,43$ \\
\hline Curtose & 0,55 & $-0,11$ & 1,02 & 0,31 \\
\hline
\end{tabular}

Tabela 1. Análise química de solo representativa (geral) para o talhão analisado

\begin{tabular}{|c|c|c|c|c|c|c|c|c|c|c|c|}
\hline \multirow{2}{*}{$\mathrm{pH}\left(\mathrm{CaCl}_{2}\right)$} & $\mathrm{H}+\mathrm{Al}$ & Al & $\mathrm{Ca}+\mathrm{Mg}$ & $\mathrm{Ca}$ & $K$ & CTC & \multirow{2}{*}{$\underset{\mathrm{mg} \mathrm{dm}}{\mathbf{P}}$} & \multirow{2}{*}{$\underset{g d^{-3}}{C}$} & \multirow{2}{*}{$\begin{array}{l}\mathbf{V} \\
\%\end{array}$} & \multirow{2}{*}{$\mathrm{Ca} / \mathrm{Mg}$} & \multirow{2}{*}{$(\mathrm{Ca}+\mathrm{Mg}) / \mathrm{K}$} \\
\hline & \multicolumn{6}{|c|}{$\mathrm{cmol}_{\mathrm{c}} \mathrm{dm}^{-3}$} & & & & & \\
\hline 5,2 & 4,96 & 0,0 & 5,8 & 4,2 & 0,16 & 10,92 & 5,3 & 21 & 55 & 2,6 & 36,3 \\
\hline
\end{tabular}

A.

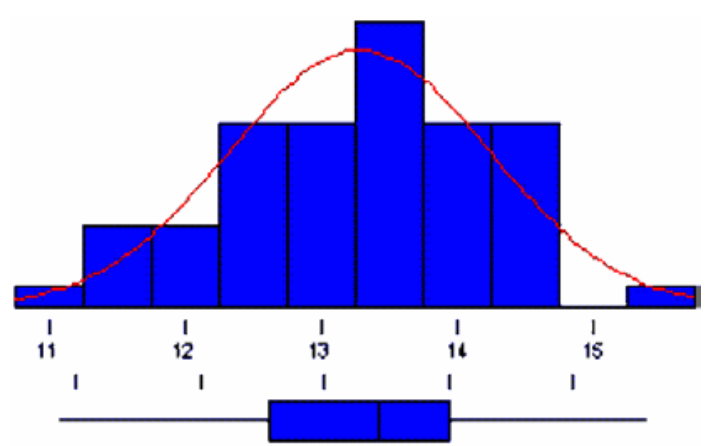

C.

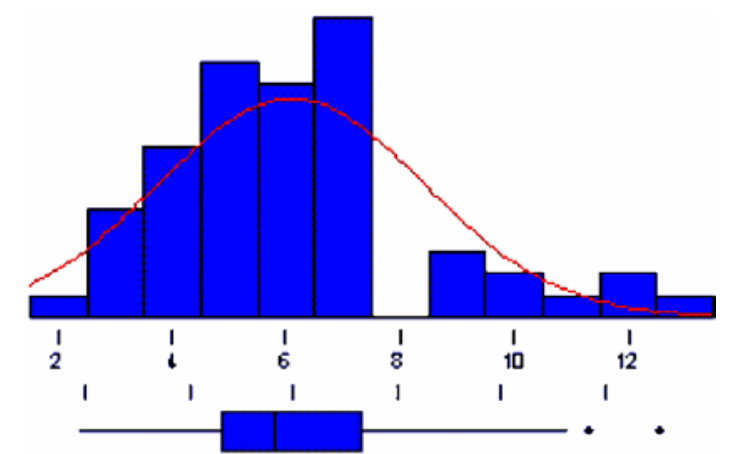

B.

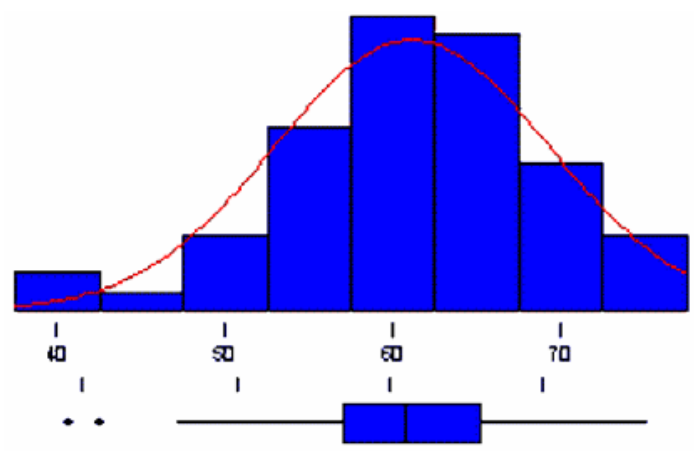

D.

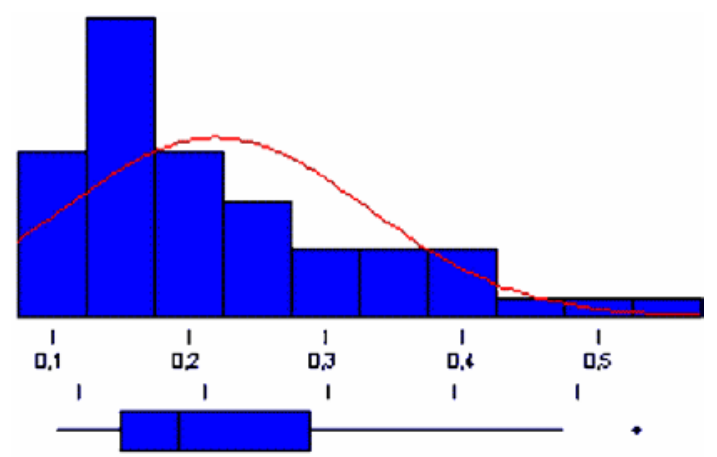

Figura 1. Histograma, curva normal e box-plot de CTC (A) $\left(\mathrm{cmol}_{\mathrm{C}} \mathrm{dm}^{-3}\right), \mathrm{V} \%(\mathrm{~B})(\%)$, fósforo (C) $\left(\mathrm{mg} \mathrm{dm}^{-3}\right)$ e potássio (D) $\left(\mathrm{cmol}_{\mathrm{C}} \mathrm{dm}^{-3}\right)$ 
A.

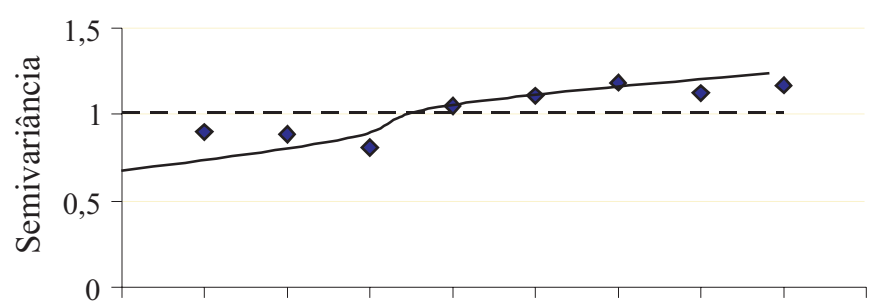

C.

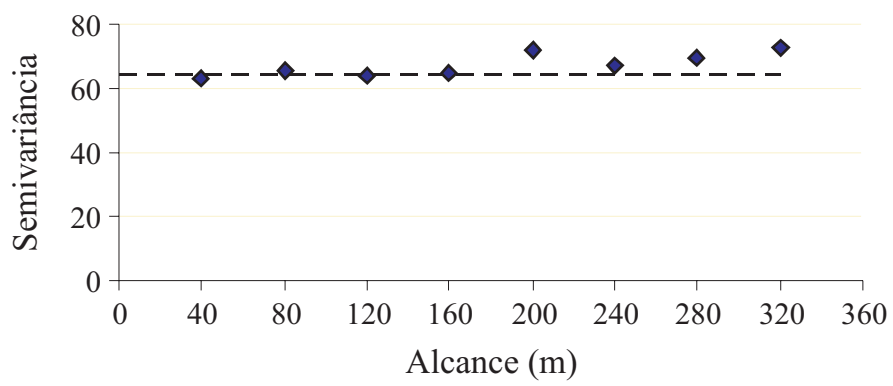

B.

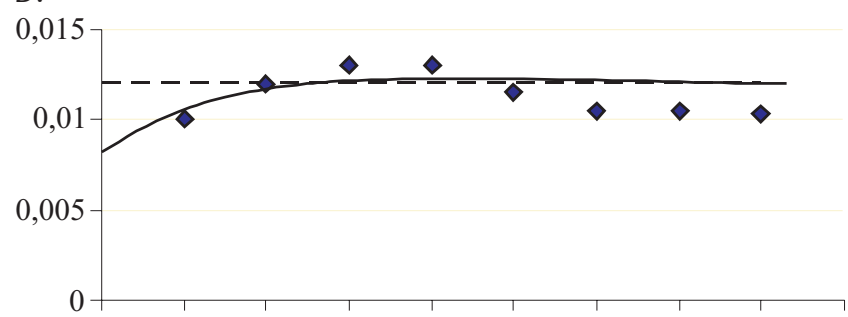

D.

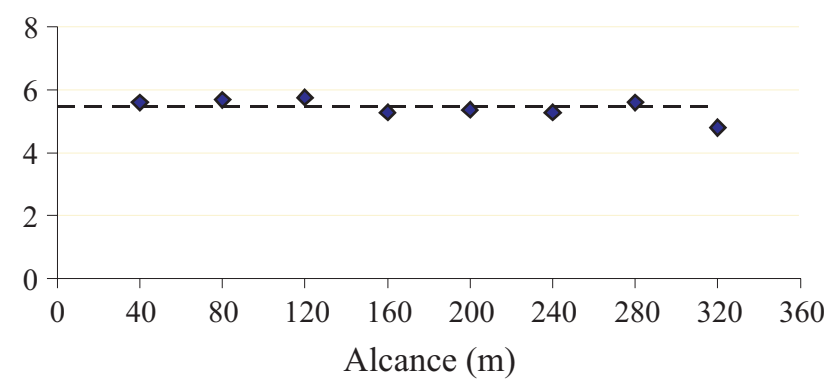

Figura 2. Semivariogramas dos dados de CTC $-\mathrm{cmol}_{C} \mathrm{dm}^{-3}(\mathrm{~A})$, potássio $-\mathrm{Cmol}_{\mathrm{C}} \mathrm{dm}^{-3}(\mathrm{~B}), \mathrm{V} \%-\%$ (C) e fósforo $-\mathrm{mg} \mathrm{dm}^{-3}$ (D)

A observação do box-plot dos dados (Figura 1) indica a presença de valores discrepantes de $\mathrm{V} \%$, fósforo e potássio, o que dificulta a utilização da análise geoestatística, pois esta se apresenta mais estável sob normalidade, segundo Silva (2000).

A análise geoestatística dos dados de CTC e do potássio, foram representados pelos semivariogramas da Figura 2 (A e B). Para os dados de CTC obteve-se modelo Gaussiano, alcance de $330 \mathrm{~m}$, patamar de 0,35 e efeito pepita de 0,84 . Para o potássio, obteve-se modelo Gaussiano, alcance de $99 \mathrm{~m}$, patamar de 0,0048 e efeito pepita de 0,0076. Para os dados de $\mathrm{V} \%$ e fósforo, não foi possível ajustar um modelo ao semivariograma, como pode ser visualizado na Figura 2 (C e D).

Os semivariogramas das variáveis V\% e fósforo, apresentaram o chamado "efeito pepita puro", em que os valores calculados se distribuem ao longo da variância, sem
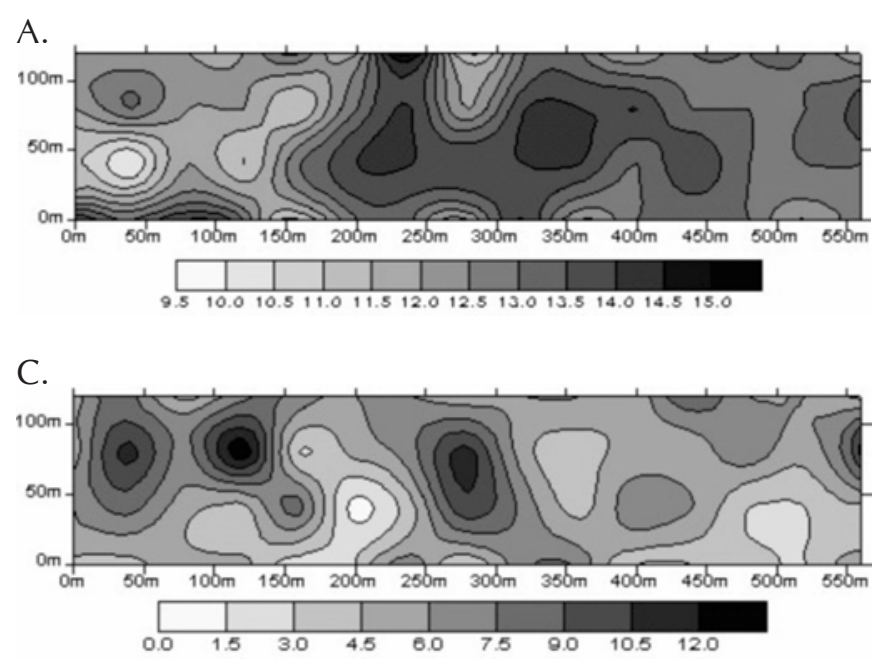

apresentar alcance definido. Este fenômeno indica ausência de dependência espacial entre amostras e, ainda, que o alcance para os dados em questão é menor que o menor espaçamento entre amostras, conforme Vieira (2000), sendo possível neste caso, apenas a aplicação da estatística clássica. Seria necessário, então, fazer-se amostragens a menores espaçamentos para se analisar essas variáveis pela geoestatística, aumentando o número de amostras.

Considerando-se a impossibilidade de se ajustar um modelo ao semivariograma de duas das variáveis estudadas, decidiu-se pela não utilização do método da krigagem na confecção dos mapas de CTC, V\%, fósforo e potássio. O método escolhido foi o da mínima curvatura, pois este apresenta acurácia e aspecto visual agradável, conforme Weirich Neto et al. (2000) e Landim (2000). Na Figura 3 é possível visualizar os mapas das variáveis utilizando-se este método.

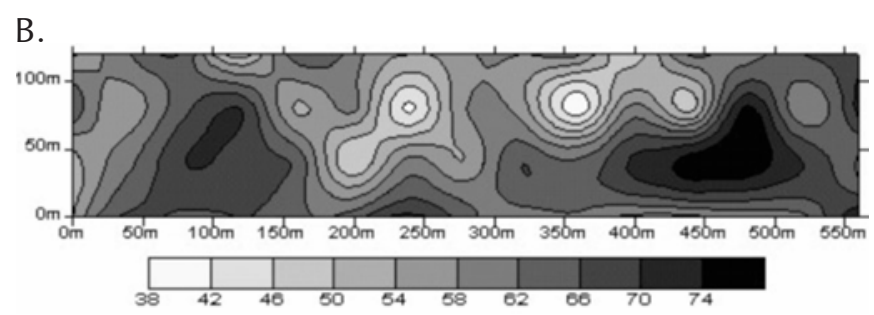

D.

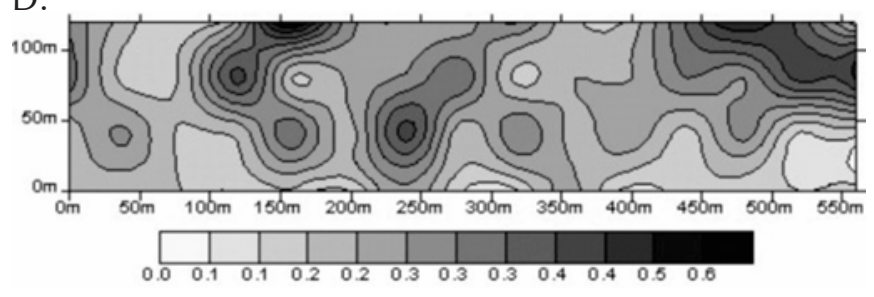

Figura 3. Mapa de valores de CTC $-\mathrm{cmol}_{\mathrm{C}} \mathrm{dm}^{-3}(\mathrm{~A}), \mathrm{V} \%-\%$ (B), fósforo $-\mathrm{mg} \mathrm{dm}^{-3}(\mathrm{C})$ e potássio $-\mathrm{cmol}_{\mathrm{C}} \mathrm{dm}^{-3}$ (D) obtidos para os 60 pontos 
Utilizando-se os dados de potássio e fósforo encontrados pela análise das amostras e as recomendações de adubação para a cultura do milho (IAC, 1997), calculou-se a formulação e a quantidade mais adequada de adubação de base para cada um dos 60 pontos. Obteve-se sete combinações de fórmulas e doses de NPK, além de três doses de uréia como adubação de cobertura, conforme visualizado nos resultados da Tabela 3 .

Em se analisando a Tabela 3, foi possível observar grandes quantidades do adubo 4-14-8, o que gera discussão; por um lado, existe a possibilidade da substituição pelo adubo 8-28-16, que permitiria que se reduzissem essas quantidades pela metade, sem alteração da fórmula; a vantagem estaria no menor valor de frete pago pela mesma quantidade de nutrientes e na maior autonomia do sistema de distribuição; por outro lado, estudos do LAMA demonstram que maiores razões de distribuição de fertilizante apresentam menores coeficientes de variação.

Pode-se visualizar, na Figura 4, a distribuição espacial das combinações de fórmulas e doses de NPK encontradas para cada ponto, conforme a descrição da Tabela 3. Na confecção do mapa da Figura 4, lançou-se mão do método denominado vizinho mais próximo, pois se desejava apenas demonstrar a distribuição dos valores encontrados, sem inferir valores intermediários a locais não amostrados.

Obtidas as fórmulas e doses para cada ponto da malha, calculou-se a diferença de fósforo e potássio entre o que foi calculado e o adubo aplicado, como se pode visualizar na Figura 5. Considerando-se pontos em que se aplicou adubo insuficiente ou em excesso, obteve-se uma diferença de $175 \mathrm{~kg}$ de $\mathrm{P}_{2} \mathrm{O}_{5}$ e -138,9 kg de $\mathrm{K}_{2} \mathrm{O}$ em 9,6 ha, indicando que, no geral, se aplicou menos fósforo e mais potássio

Tabela 3. Quantidade de uréia, combinações de fórmulas e dosagens de NPK e diferenças de fósforo e potássio entre cada combinação encontrada e a quantidade aplicada para os 60 pontos $(9,6 \mathrm{ha})$

\begin{tabular}{|c|c|c|c|c|c|}
\hline \multirow{3}{*}{$\begin{array}{c}\mathrm{N}^{0} \text { da } \\
\text { Combinação }\end{array}$} & \multirow{3}{*}{$\begin{array}{l}\text { Uréia } \\
\text { kg ha-1 }^{-1}\end{array}$} & \multirow{3}{*}{$\begin{array}{l}\text { Fórmula } \\
\text { NPK }\end{array}$} & \multirow{3}{*}{$\begin{array}{l}\text { Dose } \\
\text { de NPK } \\
\mathrm{kg} \mathrm{ha}^{-1}\end{array}$} & \multicolumn{2}{|c|}{ Diferença } \\
\hline & & & & $\mathbf{P}$ & $K$ \\
\hline & & & & \multicolumn{2}{|c|}{ kg } \\
\hline 1 & 120 & 04-14-08 & 600 & 101,18 & $-11,42$ \\
\hline 2 & 130 & $04-14-08$ & 500 & 40,83 & $-21,47$ \\
\hline 3 & 150 & $04-30-10$ & 250 & 27,07 & $-26,11$ \\
\hline 4 & 150 & $04-20-20$ & 300 & 8,45 & 4,99 \\
\hline 5 & 130 & 04-14-08 & 400 & 13,25 & $-29,09$ \\
\hline 6 & 150 & $04-14-08$ & 300 & $-7,68$ & $-45,12$ \\
\hline 7 & 120 & $20-20-20$ & 150 & $-8,06$ & $-10,66$ \\
\hline
\end{tabular}

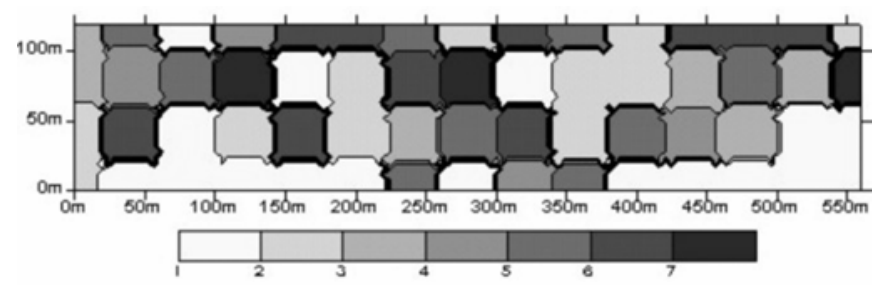

Figura 4. Mapa das combinações de fórmula e dosagem de NPK encontradas para cada ponto, conforme descrição na Tabela 3
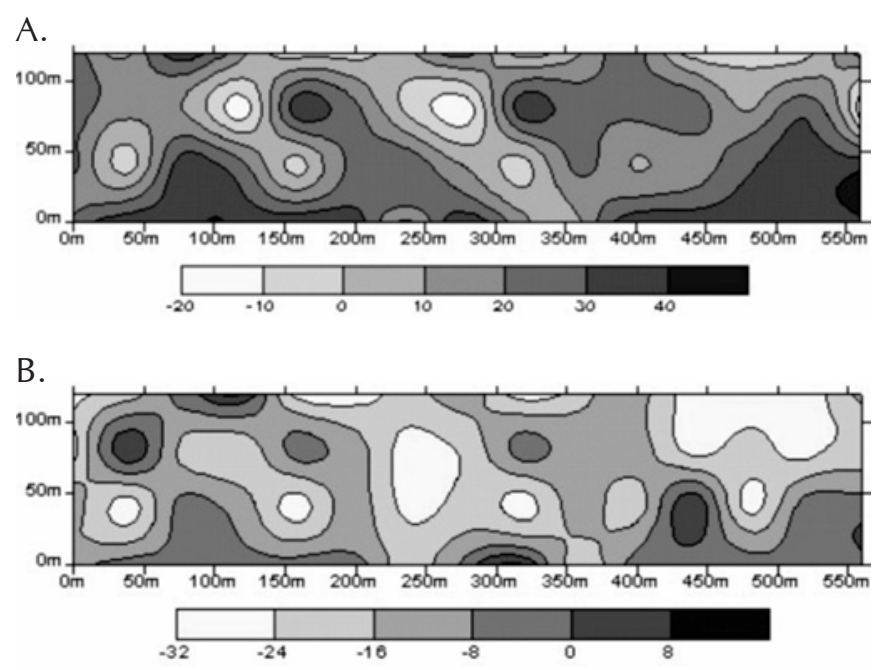

Figura 5. Diferença de fósforo (A) e potássio (B) entre a combinação de fórmula e dosagem específica por ponto e a utilizada $\left(\mathrm{kg} \mathrm{ha}^{-1}\right)$

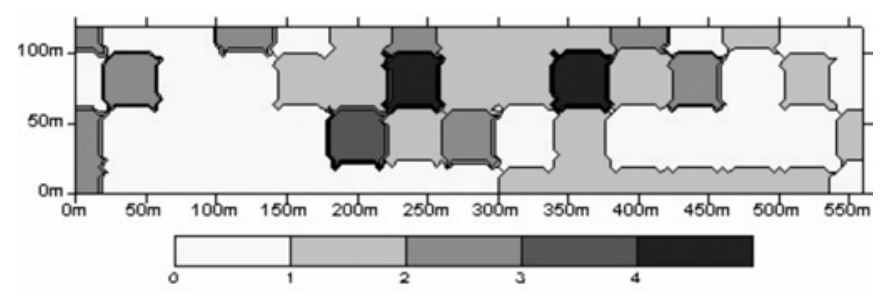

$\overline{\text { Figura 6. Mapa da necessidade de calagem para cada ponto estudado }}$ $\left(\mathrm{Mg} \mathrm{ha}^{-1}\right)$

que o necessário.

A partir dos dados de CTC e V\%, calculou-se a necessidade de calagem para cada ponto, conforme a Figura 6, a qual foi confeccionada através do método do vizinho mais próximo.

No caso específico do calcário, o técnico responsável pela área não havia programado a operação de correção da acidez do solo para o ano da realização do estudo, mas se a operação tivesse sido realizada, $7,84 \mathrm{Mg}$ de calcário deixariam de ser aplicados nos 9,6 ha, comparando-se os valores obtidos na análise das 60 amostras com os resultados da análise convencional de uma amostra de solo representativa do talhão (Tabela 1).

\section{CONCLUSÕES}

1. Observaram-se variações nas necessidades de nutrientes e de calagem entre os 60 pontos estudados, demonstrando haver diferenças dentro do talhão analisado, originalmente considerado como área homogênea.

2. Com base em diferentes sugestões de fórmulas e doses que se adaptassem a essas necessidades, pôde-se concluir que, no geral, foram aplicados $138,9 \mathrm{~kg}$ de $\mathrm{K}_{2} \mathrm{O}$ a mais que o requerido, assim como se deixou de aplicar $175 \mathrm{~kg}$ de $\mathrm{P}_{2} \mathrm{O}_{5}$ e 7,84 Mg de calcário. 


\section{LITERATURA CITADA}

Balastreire, L. A. Agricultura de precisão: mapeamento da produtividade da cultura do milho. Engenharia Rural, Piracicaba, v.8, n.1, p.97-111, 1997.

Cahn, M. D.; Hummel, J. W.; Brouer, B. H. Spatial analysis of soil fertility for site-specific crop management. Soil Science Society of America Journal, Madison, v.58, n.11, p.12401248, 1994.

EMBRAPA. Centro Nacional de Pesquisa de Solos. Sistema brasileiro de classificação de solos. Rio de Janeiro: SPI/EMBRAPA/CNPS, 1999. 412p.

Godoy, L. C. Levantamentos das potencialidades minerais do município de Ponta Grossa. Ponta Grossa: Prefeitura Municipal de Ponta Grossa. 1991. 24p.

Goering, C. E. Recycling a concept. Agricultural Engineering, St. Joseph, v.74, n.11, p.25, 1993.

IAC - Instituto Agronômico de Campinas. Recomendações de adubação e calagem para o Estado de São Paulo. van Raij, B.; Cantarella, H.; Quaggio, J. A.; Furlani, A. M. C. (ed.) $2^{\mathrm{a}}$ ed. rev. Campinas, Instituto Agronômico/Fundação IAC. 1997. 285p.

IPF - Instituto da Potassa \& Fosfato. Manual internacional de fertilidade do solo. Tradução e adaptação: Lopes, A. S., 2.ed. Piracicaba: Potafos. Título original: International soil fertility manual. 1998. 177p.

Jones, T. A. Skewness and kurtosis as criteria of normality in observed frequency distributions. Journal of Sedimentary Petrology, Salida, v.39, n.4, p.1622-1627, 1969.

Landim, P. M. B. Introdução aos métodos de estimação espacial para a confecção de mapas. Rio Claro: UNESP, Texto Didático 02, 2000. http://www.rc.unesp.br/igce/aplicada/ textodi.html. 10 Fev. 2001.

Malzer, G. L. Is Precision farming good for society? Better Crops With Plant Food, Norcross, v.80, n.3, p.6-8, 1996.

Matheron, G. Principles of geostatistics. Economic Geology, Littleton, v.58, n.8, p.1246-1266, 1963.

Mello, F. A. F.; Brasil Sobrinho, M. D. C.; Arzolla, S.; Silveira, R. I.; Cobra Neto, A.; Kiehl, J. C. Fertilidade do solo. 3.ed. São Paulo: Nobel, 1983. 400p.
Mengel, K.; Kirkby, E. A. Principles of plant nutrition. Bern: International Potash Institute, 1987. 687p.

Ndiaye, J. P.; Yost, R. S. Corn response to spatial variability of residual potassium. Soil Science, Baltimore. v.148, n.1, p.1-7, 1989.

Pannatier, Y. Variowin: Software for spatial data analysis in 2D. New York: Springer-Verlag, 1996. http://www-sst.unil.ch/research/variowin/. 7 Mar. 2000.

Reichardt, K.; Vieira, S. R.; Libardi, P. L. Variabilidade espacial de solos e experimentação de campo. Revista Brasileira de Ciência do Solo, Campinas, v.10, n.1, p.1-6, 1986.

Sá, M. F. M. Abordagem quantitativa na predição espacial de atributos do solo e geração de zonas de manejo agrícola. Piracicaba: ESALQ/USP. 2001, 117p. Tese Doutorado

SEAB - Secretaria de Agricultura e Abastecimento do Paraná. Comparativo de área, produção e produtividade - 2000/01-2001/022002/03. http://www.pr.gov.br/celepar/seab/deral. 25 Maio 2003.

Silva, E. A. A. Aplicação de um estimador robusto na análise de variabilidade espacial de um Latossolo Roxo. Cascavel: DEA/ UNIOESTE, 2000. 109p. Dissertação Mestrado

Souza, L. da S.; Cogo, N. P.; Vieira, S. R. Variabilidade de fósforo, potássio e matéria orgânica no solo em relação a sistemas de manejo. Revista Brasileira de Ciência do Solo, Campinas, v.22, n. 1, p.77-86, 1998.

Tweeten, L. Is precision farming good for society? Better Crops With Plant Food, Norcross, v. 80, n.3, p.3-5, 1996.

van Raij, B. Fertilidade do solo e adubação. São Paulo: Ed. Agronômica Ceres, Potafos, 1991.

Vieira, S. R. Variabilidade espacial de argila, silte e atributos químicos em uma parcela experimental de um Latossolo Roxo de Campinas, SP. Bragantia, Campinas, v.56, n.1, p.180-190, 1997.

Vieira, S. R. Geoestatística em estudos de variabilidade espacial do solo. In: Novais, R. F. de; Alvarez-Venegas, V. H.; Schaefer, C. E. G. R. (ed.) Tópicos em Ciência do Solo, Sociedade Brasileira de Ciência do Solo, Viçosa, v.1, p.1-53, 2000.

Weirich Neto, P. H.; Buzolini Jr, O.; Rocha, J. V. da; Borghi, E.; Sverzut, C. B. Variabilidade espacial do teor de areia, utilizando-se diferentes métodos de interpolação. In: FERTBIO, 1. Santa Maria, 2000. Anais... Santa Maria, SBCS/SBM/UFSM, 2000. CD-ROM. 